\title{
A Convolution-Free Finite-Element Time-Domain Method for the Nonlinear Dispersive Vector Wave Equation
}

\author{
David S. Abraham ${ }^{1}$, Student Member, IEEE, and Dennis D. Giannacopoulos ${ }^{1}$, Senior Member, IEEE \\ ${ }^{1}$ Department of Electrical \& Computer Engineering, McGill University, Montréal, Québec H3A 0E9, Canada
}

\begin{abstract}
In this paper, a Finite-Element Time-Domain method is presented for the solution of the second-order Vector Wave Equation (VWE) subject to electrically complex materials, including general combinations of linear dispersion, instantaneous nonlinearity, and dispersive nonlinearity. The presented method is novel in that it offers greater geometric flexibility than existing Finite-Difference methods, incorporates both instantaneous and dispersive nonlinearity, scales to arbitrary dispersive and nonlinear orders, and is simpler, faster, and requires less computational complexity than existing mixed formulations due to the use of edge elements only.
\end{abstract}

Index Terms_Dispersive Media, Nonlinear Media, Finite-Element Methods, Time-Domain Analysis

\section{INTRODUCTION}

$\mathbf{T}$ HE need for fast, efficient, and reliable telecommunications infrastructure has evolved and grown tremendously over the past decades. In particular, advances in nonlinear optics, material sciences, and fabrication methods have vastly improved data transmission and reception, and continue to be indispensable tools as devices and people become increasingly connected. As a result, the need for techniques to aid in the design of electromagnetic devices which leverage complex material interactions has also grown rapidly, representing a cost-effective substitute for empirical study.

Given this present need, several numerical methods have been devised for the study of materials exhibiting complex properties, such as dispersion and nonlinearity, however in general they tend to contain simplifications. For example, popular approximative methods such as the Slowly Varying Envelope Approximation (SVEA) [1] and the Nonlinear Schrödinger Equation (NLSE) [2], may require slowly varying structures or waveforms, cannot contend with abrupt changes in material properties, or require propagation confined to a relatively small angular area. As a result, in many cases, only a full-wave (non-approximative) treatment of the nonlinear Maxwell's Equations can yield the true behaviour of a physical system. For instance, Joseph \& Taflove [3], [4] found several instances in which the NLSE disagreed with a method which solved Maxwell's Equations directly.

One popular numerical method for Maxwell's Equations is the Finite-Element Time-Domain (FETD) method. However, the adaptation of FETD to nonlinear media has not yet received much treatment to date. FETD methods dealing with linear dispersion [5], nonlinear conductivity [6] and nonlinear permeability [7], [8] have been developed, though are not very general when it comes to arbitrary electric nonlinearity. The method in [9], in contrast, models both instantaneous and dispersive nonlinearities within the mixed FETD framework.

Manuscript received October 28th, 2018. Revised, accepted, published. Corresponding author: D. S. Abraham (email: david.abraham@mail.mcgill.ca).

Color version of one or more of the figures in this paper are available online at http://ieeexplore.ieee.org.

Digital Object Identifier (inserted by IEEE).
However, the use of the auxiliary differential equation method to model dispersion and an explicit time-marching scheme, mean the resulting method is difficult to scale to higher dispersive orders, and suffers from reduced stability.

In contrast, the mixed nonlinear FETD method presented in [10] not only models nonlinearity and dispersion, but does so using the z-transform technique, Newton-Raphson iteration, as well as an implicit Crank-Nicolson based update scheme, yielding a robust, scalable, stable, and accurate algorithm, capable of modeling very general material behaviours. In this paper, a novel successor of the method in [10] is presented. Specifically, rather than being based on a mixed FETD formulation (using both edge and face elements), the method here derived is simpler and more straightforward in that it makes use of the second-order Vector Wave Equation (VWE) formulation, requiring only the use of edge elements. Furthermore, the accuracy, stability, and scalability of the original algorithm are maintained, while allowing the use of higherorder hierarchical elements and requiring less computational resources. The accuracy and convergence of the new nonlinear VWE FETD method is then verified, and the simulation of a temporal soliton demonstrated in two dimensions.

\section{Electrically Complex Media}

In ordinary linear media, a material's polarization density vector $\vec{P}$ and the electric field $\vec{E}$ are directly proportional, with the constant of proportionality being the linear electric susceptibility $\chi^{(1)}$. However, in the case of linear dispersion, the susceptibility can be frequency-dependent, necessitating a convolution between $\chi^{(1)}$ and $\vec{E}$ in time. Moreover, nonlinear materials can see the susceptibility itself also become a function of the field strength, $|\vec{E}|$. For many materials, an adequate model for the polarization density which accounts for these interactions is given by the following:

$$
\vec{P}=\epsilon_{0} \chi^{(1)} * \vec{E}+\epsilon_{0} \chi^{(3)}\left(\alpha E^{2}+(1-\alpha) g(t) * E^{2}\right) \vec{E}
$$

in which the susceptibility $\chi^{(1)}$ models linear dispersion, $\chi^{(3)}$ an instantaneous Kerr and/or stimulated Raman nonlinearity (with the $\alpha$ term controlling their relative strengths), and $*$ denoting convolution [3]. 


\section{DERIVATION}

To begin, Faraday's and Ampère's laws are expressed as follows:

$$
\begin{aligned}
\nabla \times \frac{1}{\mu} \vec{B} & =\vec{J}+\frac{\partial \vec{D}}{\partial t} \\
\nabla \times \vec{E} & =-\frac{\partial \vec{B}}{\partial t}
\end{aligned}
$$

where the displacement field $\vec{D}$ is related to the polarization density via the constitutive relation $\vec{D}=\epsilon_{0} \vec{E}+\vec{P}$.

Combining equations (2) and (3), and eliminating the magnetic field, the second-order Vector Wave Equation (VWE) for the electric field is obtained:

$$
\nabla \times \frac{1}{\mu} \nabla \times \vec{E}+\frac{\partial^{2}}{\partial t^{2}}\left(\epsilon_{0} \vec{E}+\vec{P}\right)=-\frac{\partial \vec{J}}{\partial t}
$$

Applying a standard Finite-Element variational approach, 4) can be transformed into a semi-discrete system:

$$
\frac{\partial^{2}}{\partial t^{2}}\left(\epsilon_{L} *[\tilde{T}]\{e\}+[\hat{T}]\{e\}\right)+[S]\{e\}+\{f\}=0
$$

where $\epsilon_{L}$ is the linear part of (1), and the elemental matrices and vectors are given by:

$$
\begin{aligned}
& {[\tilde{T}]_{i j}=\int_{\Omega} \vec{W}_{i}^{(1)} \cdot \vec{W}_{j}^{(1)} d \Omega} \\
& {[\hat{T}]_{i j}=\int_{\Omega} \epsilon_{0} \chi^{(3)}\left(\alpha E^{2}+(1-\alpha) g(t) * E^{2}\right) \vec{W}_{i}^{(1)} \cdot \vec{W}_{j}^{(1)} d \Omega} \\
& {[S]_{i j}=\int_{\Omega} \frac{1}{\mu}\left(\nabla \times \vec{W}_{i}^{(1)}\right) \cdot\left(\nabla \times \vec{W}_{j}^{(1)}\right) d \Omega} \\
& \{f\}_{i}=\int_{\Omega} \frac{\partial \vec{J}}{\partial t} \cdot \vec{W}_{i}^{(1)} d \Omega
\end{aligned}
$$

with $\vec{W}^{(1)}$ representing a vector 1 -form (edge element) basis function.

At this stage, a temporal discretization must be applied to (5). Rather than using the Crank-Nicolson method as in [10], here the closely related Newmark- $\beta$ method is used instead. While the Crank-Nicolson method for mixed elements is posited to be unconditionally stable (in the linear case), Newmark- $\beta$ is provably so for $\beta=\frac{1}{4}$. Furthermore, the system in (5) is simpler due to only using one type of basis function, requiring two fewer matrices to populate and store, and no need to explicitly update the magnetic field. However, the VWE kernel does admit solutions which can lead to socalled late-time growth. Thus, for very long simulations, the mixed approach may be more advantageous, despite the added complexity and cost.

Nonetheless, equation (5) contains two complicating factors. The first is the presence of convolutions within (5) and (7), and the second is the presence of the nonlinearity within (7). In the former case, convolutions will be addressed via the $\mathrm{z}$ transform technique, whereas in the latter case a nonlinear Newton-Raphson iteration will be adopted.

\section{A. The Z-Transform Technique}

When the Newmark- $\beta$ method is applied to $[5$, the convolution associated with the linear dispersive term can be shown to take the following form:

$$
\{\mathcal{L}\}(t) \triangleq \epsilon_{L} *[\tilde{T}]\{e\}(t) .
$$

Following a technique first proposed in [5], and also applied in [10], the Laplace transform of $(10)$ can be taken. The resulting $\epsilon_{L}(s)$ can generally be written as the quotient of two $s$-dependent polynomials, while the convolution itself is transformed into a multiplication. A Möbius or bilinear transformation can then be applied, mapping from the continuous $s$-domain to the discrete $z$-domain:

$$
s \mapsto \frac{2}{\Delta t} \frac{1-z^{-1}}{1+z^{-1}}
$$

Applying this transformation to the $s$-domain version of $(10)$ results in a quotient of two $z^{-1}$ dependent polynomials, multiplied by $[\tilde{T}]\{e\}(z)$. Upon normalizing the first term in the denominator and cross multiplying, one can express the $z$-transform of the convolution as a function of $z^{-1}$. By then using the time-shifting property of the $z$-transform, and converting back to the time-domain, one can obtain an update equation for the convolution at the present time, as a function of past values:

$$
\begin{aligned}
\{\mathcal{L}\}^{n}=a_{0}[\tilde{T}]\{e\}^{n} & +\cdots+a_{p}[\tilde{T}]\{e\}^{n-p} \\
& -b_{1}\{\mathcal{L}\}^{n-1}-\cdots-b_{p}\{\mathcal{L}\}^{n-p} .
\end{aligned}
$$

Rather than implement (12) directly, a more efficient procedure can be adopted, as suggested in [5]. Instead of explicitly storing past field and convolution values, they can instead be accumulated into auxiliary variables as time marching progresses. The resulting update scheme containing the auxiliary variables $\left\{\mathcal{W}_{\alpha}\right\}^{n}$ can thus be written as follows:

$$
\begin{aligned}
\left\{\mathcal{W}_{\alpha}\right\}^{n} & =a_{\alpha}[\tilde{T}]\{e\}^{n}-b_{\alpha}\{\mathcal{L}\}^{n}+\left\{\mathcal{W}_{\alpha+1}\right\}^{n-1} & \alpha<p \\
\left\{\mathcal{W}_{\alpha}\right\}^{n} & =a_{\alpha}[\tilde{T}]\{e\}^{n}-b_{\alpha}\{\mathcal{L}\}^{n} & \alpha=p \\
\{\mathcal{L}\}^{n} & =a_{0}[\tilde{T}]\{e\}^{n}+\left\{\mathcal{W}_{1}\right\}^{n-1} &
\end{aligned}
$$

Hence, any term of the form (10) can be replaced in (5) with the equivalent update expression in (15).

A similar procedure can be adopted for the nonlinear convolution present within the $[\hat{T}]$ matrix in (7). In that case, $\mathcal{B} \triangleq g(t) * E^{2}$, and the update equations become:

$$
\begin{array}{rlrl}
\mathcal{G}_{\alpha}^{n} & =h_{\alpha}\left(E^{2}\right)^{n}-w_{\alpha} \mathcal{B}^{n}+\mathcal{G}_{\alpha+1}^{n-1} & & \alpha<p \\
\mathcal{G}_{\alpha}^{n} & =h_{\alpha}\left(E^{2}\right)^{n}-w_{\alpha} \mathcal{B}^{n} & & \alpha=p \\
\mathcal{B}^{n} & =h_{0}\left(E^{2}\right)^{n}+\mathcal{G}_{1}^{n-1} &
\end{array}
$$

Substituting this into (7) yields an expression for the $[\hat{T}]$ matrix at the current time step:

$$
\begin{aligned}
{[\hat{T}]_{i j}^{n}=} & \int_{\Omega} \epsilon_{0} \chi^{(3)}\left(\alpha\left(E^{2}\right)^{n}+\right. \\
& \left.\quad(1-\alpha)\left[h_{0}\left(E^{2}\right)^{n}+\mathcal{G}^{n-1}\right]\right) \vec{W}_{i}^{(1)} \cdot \vec{W}_{j}^{(1)} d \Omega
\end{aligned}
$$




\section{B. Nonlinear Iteration}

With a procedure in place for the convolutions, the remaining concern is the nonlinearity. As mentioned in Section II and as evident in equation (19), the presence of nonlinearity dictates that the $[\hat{T}]$ matrix is now time-dependent. This amounts to requiring that the matrices be kept within the temporal derivatives when Newmark- $\beta$ is applied. The resulting nonlinear update equation then bears a striking similarity to its linear counterpart:

$$
\begin{gathered}
\left([K]^{n+1}+\frac{\Delta t^{2}}{4}[S]\right)\{e\}^{n+1}= \\
2\left([K]^{n}-\frac{\Delta t^{2}}{4}[S]\right)\{e\}^{n}-\left([K]^{n-1}+\frac{\Delta t^{2}}{4}[S]\right)\{e\}^{n-1} \\
-\left(\left\{\mathcal{W}_{1}\right\}^{n}+2\left\{\mathcal{W}_{1}\right\}^{n-1}+\left\{\mathcal{W}_{1}\right\}^{n-2}\right) \\
\quad-\frac{\Delta t^{2}}{4}\left(\{f\}^{n+1}+2\{f\}^{n}+\{f\}^{n-1}\right)
\end{gathered}
$$

where $[K]=a_{0}[\tilde{T}]+[\hat{T}]$.

This nonlinear system of equations can be solved by nonlinear iteration or root-finding. In particular, the Newton-Raphson method can be applied:

$$
\{x\}_{(k+1)}=\{x\}_{(k)}-[J]^{-1}\{F\}_{(k)}
$$

where $k$ is the iteration number, and $[\mathrm{J}]$ is the Jacobian matrix defined as:

$$
[J]_{i j}=\frac{\partial\{F\}_{i}}{\partial\{x\}_{j}} .
$$

Since the Jacobian only takes derivatives with respect to $\{e\}^{n+1}$, only terms on the left-hand side of 20 will contribute. However, due to the remarkable similarities between the Crank-Nicolson and Newmark- $\beta$ methods initially noted in [5], this term also shares many structural similarities to that derived in [10]. As a result, it is expected that the two methods should share similar Jacobians.

Indeed, by expressing the matrix-vector product explicitly, and applying the product rule, the Jacobian can be found to be:

$$
[J]_{i j}^{n}=[K]_{i j}^{n}+\frac{\Delta t^{2}}{4}[S]_{i j}+\sum_{k} \frac{\partial[K]_{i j}^{n}}{\partial\{e\}_{j}^{n}}\{e\}_{k}^{n} .
$$

After further simplification, the last term in 23) can be written as an integral, yielding the final form of the Jacobian:

$$
\begin{aligned}
{[J]_{i j}^{n}=[K]_{i j}^{n}+\frac{\Delta t^{2}}{4}[S]_{i j}+} & \\
& \int_{\Omega} \frac{1}{E^{n}} \frac{\partial \epsilon^{n}}{\partial E^{n}}\left(\vec{W}_{i}^{(1)} \cdot \vec{E}^{n}\right)\left(\vec{W}_{j}^{(1)} \cdot \vec{E}^{n}\right) d \Omega
\end{aligned}
$$

where in the present case:

$$
\frac{\partial \epsilon^{n}}{\partial E^{n}}=2 \epsilon_{0} \chi^{(3)} E^{n}\left(\alpha+(1-\alpha) h_{0}\right)
$$

As expected, the expressions in (24) and (25) are identical to those previously obtained for the mixed method in [10].

\section{IMPLEMENTATION}

While the procedure outlined in Section III] is relatively straightforward, there are nonetheless some subtleties. For example, given that the $[K]$ and $[J]$ matrices are functions of $\{e\}$, they necessarily change with each successive NewtonRaphson iteration. As a result, both matrices must be locally recomputed and globally reassembled, not only at each time step, but at each nonlinear iteration of each time step.

Furthermore, in linear elements, the elemental matrices are generally obtainable in closed-form. However, due to the presence of $E$ within the $[K]$ and $[J]$ matrices, such closed form expressions are, in general, not obtainable. As a result, both matrices must be numerically computed using quadrature rules, within each nonlinear element. This also requires that the nonlinear convolution be known at each quadrature point.

Lastly, while the underlying Newmark- $\beta$ method is unconditionally stable for linear media, there are few general techniques available to analyze the stability of nonlinear numerical methods. While it is hoped that the unconditional stability is maintained in the nonlinear case, there is no guarantee. Nevertheless, for all numerical tests performed in the following section, no instabilities were found to occur.

\section{RESUlts}

\section{A. Convergence and Accuracy}

To test the algorithm derived above an exact solution was manufactured to which the computed one could be compared. Specifically, the current density $\vec{J}$ was selected such that the exact temporal solution for the electric field over a unit square in two dimensions was the differentiated Blackman-Harris pulse. Perfect Electric Conductor (PEC) boundary conditions were applied at each of the four domain edges.

Since the linear dispersive algorithm has already been tested in [5], the test permittivity contained only a dispersive nonlinear term $(\alpha=0)$, for which $\chi^{(1)}=2.2, \chi^{(3)}=4.1$, and $g(t)$ was selected to represent a first order Debye dispersion with a relaxation time of $\tau_{e}=10^{-9} \mathrm{~s}$.

The two solutions were then compared over progressively refined uniform meshes. The solution error was measured in both the $L_{2}$ and $L_{\infty}$ norms, and is shown in Fig. 1 versus the average mesh edge length, $h$, for $\Delta t=h / c$.

Given the basis functions used, the solution should converge globally to first order in $h$ (and therefore also in $t$ ), which is precisely the observed trend in both norms. The algorithm therefore performs as expected and with good accuracy.

\section{B. Temporal Soliton}

Having demonstrated the accuracy of the method, the algorithm was then applied to the physically significant problem of a temporal soliton propagating in a dielectric slab waveguide.

A temporal soliton is a nonlinear phenomena whereby the tendency for a pulse to become distorted under anomalous linear dispersion is counteracted by a material's nonlinearity, yielding a pulse which does not change shape as it propagates.

To demonstrate this, a dielectric slab waveguide measuring $10 \mu \mathrm{m}$ wide, by $100 \mu \mathrm{m}$ long, was simulated in two 


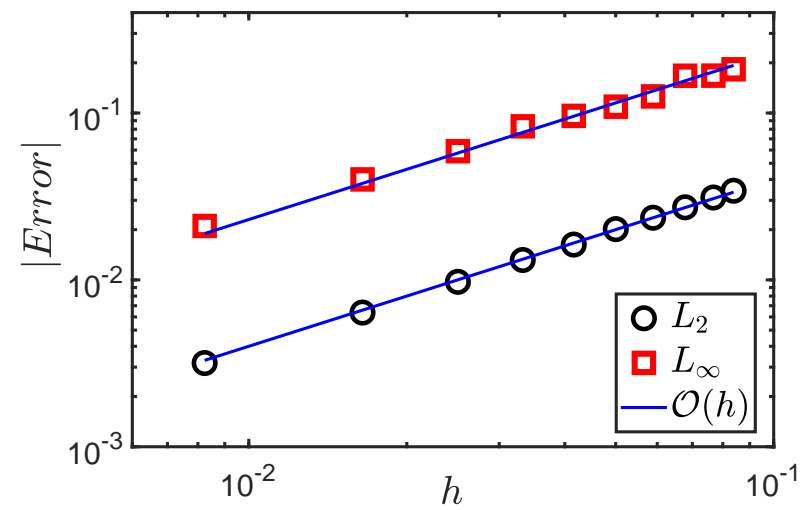

Fig. 1. Convergence in the $L_{2}$ and $L_{\infty}$ norms.

dimensions (though the derivation in Section III is independent of dimension, and thus equally applicable to problems in 3D). The center dielectric measured $2 \mu \mathrm{m}$ thick, with the remaining areas being free space. The pulse was excited on the leftmost boundary with a hyperbolic secant envelope in time. The pulse had a full width at half maximum of $52.7 \mathrm{fs}$, and a fundamental frequency of $50 \mathrm{THz}$. The linear dispersion was modeled as being second-order Lorentz, with a resonant frequency of $30 \mathrm{THz}$, damping factor of $2 \times 10^{11}$, and static/infinite susceptibilities of $6.1 \& 4.7$, respectively. The nonlinear parameters were also modeled, in part, by a Lorentztype dispersion, with an optical phonon period of $3.36 \times 10^{-14}$ $\mathrm{s}$, phonon lifetime of $1 \times 10^{-13} \mathrm{~s}, \chi^{(3)}=1.1 \times 10^{-18} \mathrm{~m}^{2} / \mathrm{V}^{2}$, and $\alpha=0.7$.

Fig. 2 shows the pulse after propagating $80 \mu \mathrm{m}$ distance within the guide. The upper plot shows the distortion experienced by the pulse in the absence of nonlinearity. In stark contrast, the introduction of nonlinearity in the bottom plot establishes a temporal soliton, whose initial shape is still roughly intact. Furthermore, a rough comparison of this result to that obtained in [10] reveals that the VWE formulation performs about $13 \%$ faster and consumes $37 \%$ less memory than the mixed method, for $h=0.3 \mu \mathrm{m}$.

\section{CONCLUSION}

In conclusion, a novel formulation of the Finite-Element Time-Domain method has been presented for the treatment of electrically complex materials. In contrast to existing techniques, the presented algorithm adopts the FETD, $z$-transform, Newmark- $\beta$, and Newton-Raphson approaches, yielding a flexible, stable, accurate, and scalable scheme. Moreover, by discretizing the vector wave equation, rather than the coupled first order Maxwell's equations, the resulting algorithm is simpler in that it only requires the use of vector edge elements. This results in fewer memory requirements, faster execution times, as well as the ability to implement higher order hierarchical basis functions. The accuracy of the method was verified via a convergence study, and the creation of an optical soliton demonstrated in a dielectric slab waveguide.

Lastly, as detailed briefly in Section IV, the presented nonlinear algorithm poses a significant computational burden.

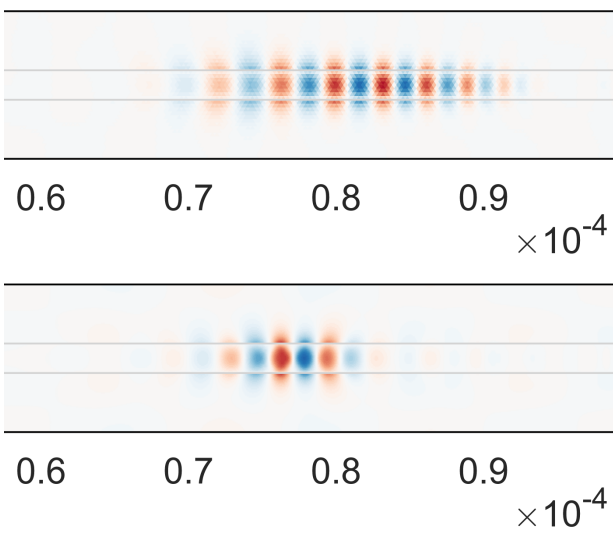

Fig. 2. Comparison of pulse distortion (top) caused by anomalous linear dispersion, and a temporal soliton (bottom).

However, there are strategies available to mitigate this cost. For example, Jacobian-Free Newton-Krylov methods [11] can be used instead of traditional Newton-Raphson iteration, alleviating the need to recompute and reassemble the Jacobian each iteration. Moreover, many aspects of the algorithm above are suitable for implementation on emerging parallel architectures, such as Graphics Processing Units (GPUs), with initial investigations showing substantial improvement.

\section{ACKNOWLEDGMENT}

The authors would like to thank the Natural Sciences and Engineering Research Council of Canada (NSERC) for their support.

\section{REFERENCES}

[1] R. Boyd, Nonlinear Optics, 3rd ed. Burlington, MA, USA: Academic Press, 2008

[2] M. F. S. Ferreira, Nonlinear Effects in Optical Fibers, 3rd ed. Hoboken, New Jersey: John Wiley \& Sons, Inc., 2011, ch. 4, pp. 63-84.

[3] R. M. Joseph and A. Taflove, "FDTD maxwell's equations models for nonlinear electrodynamics and optics," IEEE Transactions on Antennas and Propagation, vol. 45, no. 3, pp. 364-374, Mar. 1997.

[4] R. M. Joseph and A. Taflove, "Spatial soliton deflection mechanism indicated by FD-TD maxwell's equations modeling," IEEE Photonics Technology Letters, vol. 6, no. 10, pp. 1251-1254, Oct. 1994.

[5] A. Akbarzadeh-Sharbaf and D. D. Giannacopoulos, "A stable and efficient direct time integration of the vector wave equation in the finiteelement time-domain method for dispersive media," IEEE Transactions on Antennas and Propagation, vol. 63, no. 1, pp. 314-321, Jan. 2015.

[6] S. Yan and J.-M. Jin, "Time-domain finite element modeling of nonlinear conductivity using newton's method," 2015 IEEE International Symposium on Antennas and Propagation \& USNC/URSI National Science Meeting, July 2015.

[7] S. Yan and J.-M. Jin, "Theoretical formulation of a time-domain finite element method for nonlinear magnetic problems in three dimensions," Progress in Electromagnetics Research, vol. 153, pp. 33-55, 2015.

[8] S. L. Ho, Y. Zhao, W. N. Fu, and P. Zhou, "Application of edge elements to 3-d electromagnetic field analysis accounting for both inductive and capacitive effects," IEEE Transactions on Magnetics, vol. 52, no. 3, Mar. 2016.

[9] A. Fisher, D. White, and G. Rodrigue, "An efficient vector finite element method for nonlinear electromagnetic modeling," Journal of Computational Physics, vol. 225, no. 2, pp. 1331-1346, Feb. 2007.

[10] D. S. Abraham and D. D. Giannacopoulos, "A convolution-free mixed finite-element time-domain method for general nonlinear dispersive media," IEEE Transactions on Antennas and Propagation, vol. 67, no. 1, pp. 324 - 334, Jan. 2019

[11] D. A. Knoll and D. E. Keyes, "Jacobian-free newton-krylov mehotds: A survey of approaches and applications," Journal of Computational Physics, vol. 193, no. 2, pp. 357-397, 2004. 